\title{
Estimation from Relative Measurements: Error Bounds From Electrical Analogy
}

\author{
Prabir Barooah and \#João P. Hespanha \\ Dept. of Electrical and Computer Eng., Univ. of California, Santa Barbara, CA 93106 \\ Email:\{pbarooah, hespanha\} @ece.ucsb.edu
}

\begin{abstract}
We consider the problem of estimating vectorvalued variables from noisy "relative" measurements. The measurement model can be expressed in terms of a graph, whose nodes correspond to the variables being estimated and the edges to noisy measurements of the difference between the two variables associated with the corresponding nodes (i.e., their relative values). This type of measurement model appears in several sensor networks problem.

We take the value of one particular variable as a reference and consider the Unbiased Minimum Variance (UMV) estimators for the differences between the remaining variables and the reference. We establish upper and lower bounds on the estimation error variance of a node's variable as a function of the Euclidean distance in a drawing of the graph between the node and the reference one. These bounds result in a classification of graphs : civilized and dense, based on how the variance grows with distance: at a rate greater than or less than linearly, logarithmically, or bounded.

In deriving these results, we establish and exploit an analogy between the UMV estimator variance and the effective resistance in a generalized electrical network that is significant on its own.
\end{abstract}

\section{INTRODUCTION}

We consider the estimation of $n$ vector-valued variables $x_{1}, x_{2}, \ldots, x_{n} \in \mathbb{R}^{k}$ based on several noisy "relative measurements" $\zeta_{i j}$. The measurement indexes $(i, j)$ take values in some set $\mathbf{E}$ of pairs of values from $\mathbf{V}:=\{1,2, \ldots, n\}$. The term "relative" comes from the measurement model considered:

$$
\zeta_{i j}=x_{j}-x_{i}+\epsilon_{i j}, \quad \forall(i, j) \in \mathrm{E},
$$

where the $\epsilon_{i j}$ are uncorrelated zero-mean noise vectors with covariance matrices $\tilde{R}_{i j}=\mathrm{E}\left[\epsilon_{i j} \epsilon_{i j}^{\prime}\right]$. Just with relative measurements, determining the $x_{i}$ 's is only possible up to an additive constant. To avoid this ambiguity, we assume that a particular variable (say $x_{o}, o \in \mathbf{V}$ ) is used as the reference and therefore $x_{o}=0$.

The measurement equations (1) can be expressed in terms a directed graph $G=(\mathbf{V}, \mathbf{E})$ with $|\mathbf{V}|=n$ vertices (nodes) and $|\mathbf{E}|=m$ edges, with an edge between two nodes $i$ and $j$ if the measurement $\zeta_{i j}$ is available. The vector $x_{i}$ is called the ith node variable. In this paper we investigate how the structure of the graph $G$ affects the "quality" of the optimal estimate $\hat{x}_{i}$ of $x_{i}$, measured in terms of the covariance of the estimation error $\Sigma_{i}$. Optimal estimate refers to the

Best paper award at the 2nd Int. Conf. on Intelligent Sensing and Information Processing. classical Unbiased Minimum Variance Estimator (UMV), which achieves the minimum variance among all linear unbiased estimators.

We investigate asymptotic bounds on the error variance of the estimate of a node's variable as a function of the node's distance from the reference node. When $G$ is a tree, there is a single path between the $i$ th and the reference node and one can show that the covariance matrix of the estimation error is the sum of the covariance matrices associated with this path. Thus, for trees, the variance of the UMV estimation error of $x_{i}$, i.e., $x_{i}-\hat{x}_{i}$ grows linearly with the graphical distance from the $i$ th to the reference node. By the graphical distance between two nodes we mean the minimum number of edges one has to traverse in going from one node to the other. It turns out that for graphs "denser" than trees, the variance of the UMV estimation error can grow less than linearly with distance.

The graph theoretical concept of drawing allows one to develop a suitable notion of graph "density/sparsity." The drawing of a graph is simply a function that maps its nodes to points in $\mathbb{R}^{d}, d \geq 1$. A graph is said to be civilized ${ }^{1}$ if its nodes can be drawn in $\mathbb{R}^{d}$ such that (i) there is a minimum distance between every two nodes and (ii) there is a maximum distance $r$ between nodes connected by an edge. Intuitively, the nodes and edges of civilized graph are "sufficiently sparse to be drawn in $\mathbb{R}^{d}$ without too much clutter." A graph is said to be dense in $\mathbb{R}^{d}$ if its nodes can be drawn in $\mathbb{R}^{d}$ such that (i) every ball in $\mathbb{R}^{d}$ with diameter $\gamma$ contains at least one node and (ii) any two nodes with distance smaller than $\rho$ are connected by an edge. Intuitively, dense graphs with sufficiently large $\rho$ compared to $\gamma$ have "sufficiently many nodes and edges to cover $\mathbb{R}^{d}$ without holes." The two concepts above allow one to characterize precisely how the variance of the UMV estimation error grows with distance. Table I summarizes the main results of this paper.

A crucial step in proving the results in Table I, is to establish that the covariance matrices of the UMV estimation errors are numerically equal to effective resistance in a suitably defined electrical circuit. Such equivalence was first noted by Karp et al. [7] for scalar measurements $(k=1)$.

\footnotetext{
${ }^{1}$ Civilized graphs appeared in Doyle and Snell [3] in connection with random walks, but with the terminology " $G$ can be drawn in a civilized manner in $\mathbb{R}^{d "}$ " that we shorten to " $G$ is civilized in $\mathbb{R}^{d "}$."
} 
TABLE I

VARIANCE $\Sigma_{u, o}$ OF THE UMV ESTIMATION ERROR OF $x_{u}$ WITH RESPECT TO THE REFERENCE $x_{o}=0$ VERSUS THE EUCLIDEAN DISTANCE $d_{f}(u, o)$ BETWEEN THE DRAWING OF $u$ AND $o$. FOR FUNCTIONS $g$ AND $p$, WhERE $g, p: \mathbb{R} \rightarrow \mathbb{R}^{k \times k}$, AND $p: \mathbb{R} \rightarrow \mathbb{R}$, THE NOTATION $g(y) \geq \Omega(p(y)) I_{k}$ MEANS THAT THERE EXISTS POSITIVE SCALARS $y_{o}, \alpha$ SUCH THAT $g(y) \geq \alpha p(y) I_{k} \quad \forall y>y_{o}$, AND $g(y) \leq \mathcal{O}(p(y)) I_{k}$ MEANS THAT $\exists y_{o}, \alpha>0$ SUCH THAT $g(y) \leq \alpha p(y) I_{k} \quad \forall y>y_{o}$.

\begin{tabular}{l|r|l} 
& Graph civilized in $\mathbb{R}^{d}$ & Graph dense in $\mathbb{R}^{d}$ with $\rho>2 \gamma$ \\
\hline$d=1$ & $\Omega\left(d_{f}(u, o) / r\right) I_{k} \leq \Sigma_{u, o}$ & $\Sigma_{u, o} \leq \mathcal{O}\left(d_{f}(u, o) / \gamma\right) I_{k}$ \\
$d=2$ & $\Omega\left(\log \left(d_{f}(u, o) / r\right)\right) I_{k} \leq \Sigma_{u, o}$ & $\Sigma_{u, o} \leq \mathcal{O}\left(\log \left(d_{f}(u, o) / \gamma\right)\right) I_{k}$ \\
$d=3$ & $\Omega(1) I_{k} \leq \Sigma_{u, o}$ & $\Sigma_{u, o} \leq \mathcal{O}(1) I_{k}$
\end{tabular}

Here, we prove that this also holds for vector measurements, provided that one considers a generalized electric circuits in which currents and voltages are vectors and resistances are matrices.

\section{A. Relevance to Sensor Networks}

This estimation problem addressed in this paper has multiple applications in the area of sensor networks. Some of these are summarized below:

Position estimation: suppose that in a region where a sensor network is deployed, there are $n$ "nodes" whose positions we want to estimate. These nodes can be events/objects/sensors whose unknown positions are of interest. We assume that every sensor is capable of measuring relative positions (with some error) between itself and its neighboring nodes. Relative position measurements can be obtained from measurements of range and bearing, provided sensors have on-board compasses so that their local coordinate systems have the same orientation, differing only in the shifts of the origins. Measurements of range and bearing can be done using RF techniques such as RSS, TDOA, TOA and AOA $[2,6]$. We are of course assuming that each node does not have, or does not want to rely upon, GPS. In this problem, $k=2$ if the nodes are located in a plane and $k=3$ if they are located in three-dimensional space.

Time synchronization: Suppose that the internal clocks of $n$ sensor nodes exhibit time shifts $x_{1}, x_{2}, \ldots, x_{n}$ with respect to a reference clock. A pair of neighboring sensors can measure their clock offsets with some error, by transmitting their local clock times to each other, resulting in a measurement model of the form eq. (1) [7]. The goal would then be to estimate the time-shifts between all nodes and a reference clock to synchronize the clocks of all $n$ nodes.

Motion consensus: Suppose that $n$ mobile nodes are moving in the plane with unit velocity and directions specified by angles $x_{i}$. Each node is only able to measure, with some error, the difference in angle of its own heading with respect to the headings of their neighboring agents. The goal is to estimate the headings of the agents in a consistent coordinate system and use that to reach a consensus in the direction of motion [4].

For most problems that arise in the context of sensor networks there are "natural drawings" that are physically meaningful and for which one would expect the graphs to be civilized and/or dense in appropriate Euclidean spaces.
Indeed, in all the examples discussed above, the variables $x_{i}$ refer to properties of objects, so the measurement graph $G$ could be "drawn" by associating to each node the Cartesian position of the corresponding object (either in $\mathbb{R}, \mathbb{R}^{2}$, or $\mathbb{R}^{3}$ depending on the problem). Such a drawing would be civilized as long as (i) there is a minimum distance between each two objects and (ii) the sensors used to obtain the relative measurements $\zeta_{i j}$ have limited range. In this case, the formulas in the second column of Table I provide explicit lower-bounds on the variance of the estimation error for $x_{u}$ as a function of the distance between the corresponding object and the reference one. Note that even if the objects are in $3 \mathrm{~d}$-space, it may be possible to obtain a civilized drawing in $\mathbb{R}^{2}$ (or even $\mathbb{R}$ ) provided that they essentially lie in a 2-D surface (or in a line).

The natural drawing considered above would be dense if (i) every ball in with a given diameter $\gamma$ contains at least one object and (ii) a relative measurement is available for every two objects with distance smaller than $\rho$. In this case, the formulas in the third column of Table I provide upperbounds on the variances of the estimation errors. Formally, dense graphs must be infinite. However, in practice all that matters is that both the reference object and the object whose variable one wants to estimate fall spatially within the remaining objects.

\section{B. Related Work}

Among the applications cited above, the problem of node position estimation has received considerable attention in recent times. The problem formulation usually assumes the availability of relative range measurements and the existence of a small number of "beacons" that precisely know their own positions (by use of GPS etc.)[10], or the availability of range and angle measurements with beacons that know their locations imprecisely [9]. When the relative measurements considered are distances and the measurement model becomes nonlinear. For this case, both Maximum Likelihood [10] and Maximum A-Posteriori [9] estimators have been considered, resulting in a nonlinear optimization problem. In time synchronization, quite a lot of work has been done on the algorithmic side, but only Karp et, al. considered the UMV estimator with a measurement model of the type given by eq. (1). In motion consensus, the emphasis so far has been in deriving control laws to achieve a certain flocking behavior assuming relative 
angular position measurements [5], but not on estimation of headings from relative angular position measurements.

To the best of our knowledge, the effect of the graph structure on the estimation accuracy has not been studies before for any of the applications mentioned.

The remainder of the paper is organized as follows: In section II, we provide the complete problem formulation and review the UMV estimator. In section III we describe the analogy between the UMV estimator covariance matrix and effective resistance in a suitably defined generalized electrical network. We also establish bounds on the effective resistance bounds for special graphs and other useful tools needed in subsequent sections. In Section IV we discuss graph drawings and formally introduce the concepts of dense and civilized graphs. The main results of the paper are stated and proved in Section V. Section VI contains some final conclusions and directions for future work.

\section{UMV ESTIMATION FROM RELATIVE MEASUREMENTS}

Let $X$ be a vector in $\mathbb{R}^{(n-1) k}$ obtained by stacking together all the unknown node variables $x_{i}, i \in \mathbf{V} \backslash o$, i.e., $X:=\left[x_{1}^{T}, x_{2}^{T}, \ldots, x_{o-1}^{T}, x_{o+1}^{T}, \ldots, x_{n}^{T}\right]^{T}$. Let the edges of the graph are $\mathbf{E}=\left\{e_{1}, e_{2}, \ldots e_{m}\right\}$, then define $Z:=$ $\left[\zeta_{e_{1}}^{T}, \zeta_{e_{2}}^{T}, \ldots, \zeta_{e_{m}}^{T}\right]^{T} \in \mathbb{R}^{k m}$ and $\Upsilon:=\left[\epsilon_{e_{1}}^{T}, \epsilon_{e_{2}}^{T}, \ldots, \epsilon_{e_{m}}^{T}\right]^{T} \in$ $\mathbb{R}^{k m}$. Eq. (1) can now be rewritten as follows:

$$
Z=\mathcal{H} X+\Upsilon
$$

where $\mathcal{H}$ is an appropriately defined measurement matrix. The covariance matrix of the measurement error vector $\Upsilon$ is a symmetric positive definite block diagonal matrix which has the edge error covariances along the diagonal: $\tilde{\mathcal{R}}:=$ $E\left[\Upsilon \Upsilon^{T}\right]=\operatorname{diag}\left(\tilde{R}_{e_{1}}, \tilde{R}_{e_{2}}, \ldots, \tilde{R}_{e_{m}}\right) \in \mathbb{R}^{k m \times k m}$

We seek an estimate for $X$ as a linear combination of the available measurements $Z$ that yields an unbiased estimate and achieves minimum estimator error variance. The solution to this problem is the Unbiased Minimum Variance (UMV) Estimator. For the measurement model (2), the UMV estimate of $X$ is given by [8]

$$
\widehat{X}=\left(\mathcal{H}^{T} \tilde{\mathcal{R}}^{-1} \mathcal{H}\right)^{-1} \mathcal{H}^{T} \tilde{\mathcal{R}}^{-1} Z
$$

and the covariance $\boldsymbol{\Sigma}$ of the estimation error is given by

$$
\boldsymbol{\Sigma}:=\mathrm{E}\left[(X-\widehat{X})(X-\widehat{X})^{T}\right]=\left(\mathcal{H}^{T} \tilde{\mathcal{R}}^{-1} \mathcal{H}\right)^{-1}
$$

For a connected graph, the estimation error covariance matrix in (4) always exists and is of finite norm [1].

\section{ANAlOGY With ElECTRICAL NETWORK}

To establish our main result we will use the fact that the error variance of the UMV estimator is equivalent to the effective resistance in an appropriately defined generalized resistive electrical network where edge currents and potential drops are vectors in $\mathbb{R}^{k}$ (called generalized currents and generalized potential drops) and resistances are matrices in $\mathbb{R}^{k \times k}$ (called generalized resistances).
A generalized electrical network consists of a connected graph $G=(\mathbf{V}, \mathbf{E})$ together with a matrix-resistance function $R: \mathbf{E} \rightarrow \mathbb{R}^{k \times k}$ such that $R(e)=R(e)^{T}>0, \forall e \in \mathbf{E}$. For such a network, a flow from node $p \in \mathbf{V}$ to node $q \in \mathbf{V}$ with vector-intensity $J \in \mathbb{R}^{k}$ is a function $j: \mathbf{E} \rightarrow \mathbb{R}^{k}$ with the property that

$$
\sum_{\substack{(u, v) \in \mathbf{E} \\ u=\bar{u}}} j_{u, v}-\sum_{\substack{(v, u) \in \mathbf{E} \\ u=\bar{u}}} j_{v, u}= \begin{cases}J & \bar{u}=p \\ -J & \bar{u}=q \\ 0 & \text { otherwise }\end{cases}
$$

$\forall \bar{u} \in V$; and a current from $p \in \mathbf{V}$ to $q \in \mathbf{V}$ with vectorintensity $I \in \mathbb{R}^{k}$ is a flow $i: \mathbf{E} \rightarrow \mathbb{R}^{k}$ for which there exists a function $V: \mathbf{V} \rightarrow \mathbb{R}^{k}$ such that

$$
R_{u, v} i_{u, v}=V_{u}-V_{v}, \quad \forall(u, v) \in \mathrm{E} .
$$

The function $V$ is called a potential associated with $i$.

Theorem 1 (Linearity): [1] Assume that $G$ is connected when regarded as an undirected graph and that $R(e)>0$, $\forall e \in \mathbf{E}$. For a given vector-intensity $I \in \mathbb{R}^{k}$, there is a unique current $i$ from $p \in \mathbf{V}$ to $q \in \mathbf{V}$ and the corresponding potential $V$ is also unique when one enforces the normalization constraint $V_{q}=0$. Moreover, $i$ and $V$ are linear functions of $I$.

Since $V$ is a linear function of the vector intensity $I$ from $p \in \mathbf{V}$ to $q \in \mathbf{V}$, for each $v \in \mathbf{V}$, there must exist a matrix $R_{p, v ; q}^{\text {cross }}$ such that

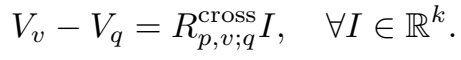

We call such matrix the cross matrix-resistance from $p$ to $v$ with respect to $q$. When $p=v$, we call it the effective matrix-resistance from $p$ to $q$, i.e.,

$$
V_{p}-V_{q}=R_{p, q}^{\mathrm{eff}} I, \quad \forall I \in \mathbb{R}^{k},
$$

For $k=1$, generalized electrical networks are the usual electrical networks with scalar currents, potentials, and resistors.

From the measurement graph $G=(\mathbf{V}, \mathrm{E})$, we form the generalized electrical network $N(G)=(\overline{\mathbf{V}}, \overline{\mathrm{E}}, R)$ in the following way. $N(G)$ has the same nodes and edges as in $G$, and the generalized electrical resistance $R(\bar{e})$ of every edge $\bar{e} \in \overline{\mathrm{E}}$ is set equal to the covariance matrix $\tilde{R}(e)$ of the measurement error vector associated with the corresponding edge $e \in \mathrm{E}$.

The following is the main result of this section:

Theorem 2: Consider an generalized electrical network $N(G)=(\overline{\mathbf{V}}, \overline{\mathrm{E}}, R)$ constructed from the measurement graph $G=(\mathbf{V}, \mathbf{E})$ with matrix-resistances $R(\bar{e})$ assigned as described above. Then,

1) The covariance matrix of the estimation error of $x_{u}$ is numerically equal to $R_{u, o}^{\mathrm{eff}}$, where $o$ is the reference node.

2) The covariance matrix between the estimation errors of $x_{u}$ and $x_{v}$ is numerically equal to $R_{u, v ; o}^{\text {cross }}$, where $o$ is the reference node. 
The proof is omitted for lack of space; the interested reader may consult [1]. A proof of the first statement of the theorem above, for the special case $k=1$ was provided by Karp et. al. [7], who considered the problem of estimating time differences among clocks in a network of sensors. Theorem 2 above provides an electrical analogy also for the covariances and more importantly, generalizes this result to estimation of vector valued parameters.

\section{A. Effective Resistance Bounds for Lattices}

With the equivalence between effective resistance and UMV estimator variance established, we present bounds on effective resistances for lattices and their $h$-fuzzes (to be defined soon) and a few key results relevant for the analysis of generalized electrical networks. These results will be useful in establishing variance bounds in the subsequent sections. Proofs are omitted for lack of space, the interested reader may consult [1]. For the purpose of brevity, from now on we refer to generalized resistances as simply resistances.

We recall that a graph $G=(\mathbf{V}, \mathbf{E})$ can be embedded in another graph $\bar{G}=(\overline{\mathbf{V}}, \overline{\mathbf{E}})$, if there is an injective map $\eta$ : $\mathbf{V} \rightarrow \overline{\mathbf{V}}$ such that $(\eta(u), \eta(v)) \in \overline{\mathbf{E}}$ for every $(u, v) \in \mathbf{E}$. The map $\eta$ is called the embedding.

Theorem 3 (Generalized Rayleigh's Monotonicity Law):

[1] Consider two generalized electrical networks with graphs $G=(\mathbf{V}, \mathbf{E})$ and $\bar{G}=(\overline{\mathbf{V}}, \overline{\mathbf{E}})$ and matrix-resistances $R$ and $\bar{R}$, respectively. If $G$ can be embedded in $\bar{G}$ with an embedding $\eta$ and $R(e) \geq \bar{R}(\eta(e)), \forall e \in \mathbf{E}$, then

$$
R_{p, q}^{\mathrm{eff}} \geq \bar{R}_{\eta(p), \eta(q)}^{\mathrm{eff}}, \quad \forall p, q \in \mathbf{V} .
$$

Now we formally define lattices. A $d$-D lattice in $\mathbb{R}^{d}$ is a graph that has a vertex at every point in $\mathbb{R}^{d}$ with integer coordinates and an edge between every two vertices with an Euclidean distance of 1 between them. We denote the $d$-D lattice in $\mathbb{R}_{d}$ by $\mathbf{Z}_{d}$.

For any integer $h$, the $h$ - fuzz $G^{(h)}$ of a graph $G$ is the graph obtained from $G$ by adding an edge $(u, v)$ whenever the graphical distance between the nodes $u$ and $v$ is less than or equal to $h$ [3].

Theorem 4: [1] Consider the electrical network $N\left(\mathbf{Z}_{d}^{(h)}\right)$, with equal generalized resistance $R(e)=$ $R(e)^{T}>\mathbf{0}$ for every edge $e$ of $\mathbf{Z}_{d}^{(h)}$. We denote by $d_{\mathbf{Z}_{d}}(u, v)$ the graphical distance between two nodes $u$ and $v$ in the lattice $\mathbf{Z}_{d}$. The effective resistance $R_{u, v}^{\text {eff }}$ between two nodes $u$ and $v$ in $\mathbf{Z}_{d}^{(h)}$ satisfies the following relations:

1) For $d=1, R_{u, v}^{\mathrm{eff}}\left(d_{\mathbf{Z}_{1}}(u, v)\right)=\Theta\left(\frac{d_{\mathbf{z}_{1}}(u, v)}{h}\right) R,{ }^{2}$

2) For $d=2, R_{u, v}^{\mathrm{eff}}\left(d_{\mathbf{Z}_{2}}(u, v)\right)=\Theta\left(\log \left(\frac{d_{\mathbf{Z}_{2}}(u, v)}{h}\right)\right) R$.

3) For $d=3, R_{u, v}^{\text {eff }}\left(d_{\mathbf{Z}_{3}}(u, v)\right)=\Theta(1) R$.

\section{DRAWING GRAPHS}

An Euclidean drawing function for a graph $G=(\mathbf{V}, \mathrm{E})$ is a function $f: \mathbf{V} \rightarrow \mathbb{R}^{d}, d \geq 1$. Given two nodes $u, v \in$ $\mathbf{V}$ we call $d_{f}(u, v):=\|f(v)-f(u)\|$ the Euclidean distance

\footnotetext{
${ }^{2} g(y)=\Theta(p(y)) I_{k}$ means that $g(y) \geq \Omega(p(y)) I_{k}$ and $g(y) \leq$
} $\mathcal{O}(p(y)) I_{k}$. between them induced by $f$. Drawing functions can be used to "measure" the density of nodes and edges in a graph as follows.

For a pair $(G, f)$, the minimum node distance is defined by

$$
s:=\inf _{\substack{v, u \in V \\ v \neq u}} d_{f}(u, v)
$$

and the maximum connected range by

$$
r:=\sup _{(u, v) \in E} d_{f}(u, v) .
$$

A graph $G$ can be drawn in a civilized manner in Euclidean space $\mathbb{R}^{d}$ if there exists a drawing function $f: \mathbf{V} \rightarrow \mathbb{R}^{d}$ with a positive minimum node distance and finite maximum edge length.

Intuitively, the nodes and edges of civilized graph are "sufficiently sparse to be drawn in $\mathbb{R}^{d}$ without too much clutter." The definition above is essentially a refinement of the definition given by Doyle and Snell [3], with the quantities $r$ and $s$ made to assume precise values for a particular drawing. For short, we say the graph $G$ is civilized in $\mathbb{R}^{d}$ if it can be drawn in a civilized manner in $\mathbb{R}^{d}$. Any finite graph is civilized in $\mathbb{R}^{d}, \forall d \geq 1$ but the same is not true for infinite graphs. For example, an infinite 2-D lattice is only civilized in $\mathbb{R}^{d}, \forall d \geq 2$ whereas an infinite 3 -D lattice is only civilized in $\mathbb{R}^{d}, \forall d \geq 3$. Note that there is no requirement of edges not intersecting. It follows from the definition that a civilized graph is always of bounded degree.

For a pair $(G, f)$, the minimum disconnected range is defined by

$$
\rho:=\inf _{\substack{(u, v) \neq E \\ v, u \in V, v \neq u}} d_{f}(u, v) .
$$

and the maximum uncovered diameter $b^{3}$

$$
\gamma:=\sup \left\{\delta: f(V) \cap B_{\delta} \neq \emptyset, \forall B_{\delta}\right\}
$$

where the universal quantification spans over all balls $B_{\delta}$ with diameter $\delta$. Note that $\gamma$ is the diameter of the largest open ball that can be placed in $\mathbb{R}^{d}$ not containing the drawing of any node, and $\rho$ is the maximum range that if the two nodes are within a Euclidean distance $\rho$ of each other in the drawing of $G$, they are guaranteed to have an edge between them in $G$.

A graph $G$ is dense in $\mathbb{R}^{d}$ if there exists a drawing function $f$ with positive minimum disconnected range $\rho$ and finite maximum uncovered diameter $\gamma$.

Intuitively, dense graphs have "sufficiently many nodes and edges to cover $\mathbb{R}^{d}$ without holes." A finite graph can never be dense in $\mathbb{R}^{d}, d \geq 1$; an infinite 2 -D lattice is only

\footnotetext{
${ }^{3}$ The definition in eq. (7) could also be written as $s:=\sup \{\delta: \mid f(V) \cap$ $\left.B_{\delta} \mid \leq 1, \forall B_{\delta}\right\}$, and the definition in eq. (9) as $\gamma:=\inf \{\delta: \mid f(V) \cap$ $\left.B_{\delta} \mid \geq 1, \forall B_{\delta}\right\}$, which emphasizes their parallelism.
} 
dense in $\mathbb{R}^{d}, d \leq 2$; and an infinite 3 -D lattice is only dense in $\mathbb{R}^{d}, d \leq 3$.

It turns out that graphs that are civilized/dense in $\mathbb{R}^{d}$ can be bounded by $d$-D lattices with respect to the partial order induced by the embedding relation.

Lemma 1: [1] If $G=(\mathbf{V}, \mathrm{E})$ is civilized in $\mathbb{R}^{d}$ then $G$ can be embedded in the $h$-fuzz of a $d$-D lattice with $h:=\left\lceil\frac{d(r+s)}{s}\right\rceil$, where $s>0$ and $r<\infty$ are the minimum node distance and maximum connected range, respectively, for some Euclidean drawing $f$ of $G$. Moreover, $\forall u, v \in \mathbf{V}$,

$$
\sqrt{d}\left(\frac{d_{f}(u, v)}{s}-1\right) \leq d_{\mathbf{Z}_{d}}(\eta(u), \eta(v))
$$

where $\eta$ denotes the embedding and $d_{\mathbf{z}_{d}}$ the graphical distance in the $d$-D lattice $\mathbf{Z}_{d}$.

Lemma 2: [1] If $(\mathbf{V}, \mathrm{E})$ is dense in $\mathbb{R}^{d}$ with minimum disconnected range $\rho>0$ and maximum uncovered diameter $\gamma<\infty$ satisfying $2 \gamma<\rho$ for some Euclidean drawing function $f$, then the $d$-D lattice can be embedded in $G$. Moreover, $\forall u, v \in \mathbf{V}$,

$$
d_{\mathbf{Z}_{d}}(\eta(u), \eta(v)) \leq \sqrt{d}\left(\frac{d_{f}(u, v)}{\gamma}+1\right)
$$

where $\eta$ denotes the embedding and $d_{\mathbf{z}_{d}}$ the graphical distance in the $d$-D lattice $\mathbf{Z}_{d}=(\overline{\mathbf{V}}, \overline{\mathrm{E}})$.

\section{VARIANCE VS. DistancE FOR CIVILIZED AND DENSE GRAPHS IN $\mathbb{R}^{d}$}

For graphs that can be drawn in a civilized/dense manner in $\mathbb{R}^{d}$ with some drawing function $f$, we are able to bound the UMV estimator variance $\Sigma_{u, o}:=\mathrm{E}\left[\left(x_{u}-\hat{x}_{u}\right)\left(x_{u}-\right.\right.$ $\left.\hat{x}_{u}\right)^{T}$ ] of a node variable $x_{u}$ as a function of the Euclidean distance between node $u$ and the reference node $o$ induced by $f$. When measurement error covariance matrices are of bounded norm, for civilized graphs we can establish lower bounds and for dense graphs with $\rho \geq 2 \gamma$, upper bounds. The two theorems presented here are the main results of the paper. Again, "resistance" is used to denote generalized resistance for brevity.

Let $G$ be a connected graph when it is regarded as undirected and let $\Sigma_{u o}$ denote the UMV estimator variance of $x_{u}$ 's estimate with node $o$ as the reference. Suppose that the maximum and minimum eigenvalues of the measurement error covariance matrices associated with the edges in $G$ are upper and lower bounded between two positive numbers $\sigma_{\text {max }}^{2}$ and $\sigma_{\text {min }}^{2}$, respectively.

Theorem 5: If $G$ is civilized in $\mathbb{R}^{d}, 1 \leq d \leq 3$, and the conditions stated above are satisfied, the following statements hold:

1) If $G$ can be drawn in a civilized manner in $\mathbb{R}$ with some drawing function $f$, then $\Sigma_{u, o} \geq$ $\Omega\left(d_{f}(u, o) / r\right) \sigma_{\min }^{2} I_{k}$.

2) If $G$ is civilized in $\mathbb{R}^{2}$, then $\Sigma_{u, o} \geq$ $\Omega\left(\log \left(d_{f}(u, o) / r\right)\right) \sigma_{\min }^{2} I_{k}$.

3) If $G$ is civilized in $\mathbb{R}^{3}$, then $\Sigma_{u, o} \geq \Omega(1) \sigma_{\min }^{2} I_{k}$.
Proof. We will prove the second statement (for $\mathbb{R}^{2}$ ). The statements for graphs that are civilized in $\mathbb{R}$ and $\mathbb{R}^{3}$ can be proved in a similar way.

From the equivalence of effective-resistance and estimator variance (theorem 2), we know that $\Sigma_{u, o}$ is equal to the generalized effective resistance between nodes $u$ and $o$ in the electric network $N(G)$ where the generalized resistances on the edges are set equal to the measurement error variances associated with the corresponding edges in $G$. Since $G$ (and therefore $N(G)$ ) can be drawn in a civilized manner in $\mathbb{R}^{2}$, it can be embedded in the $h_{o^{-}}$ fuzz of the 2-D lattice, $\mathbf{Z}_{d}^{\left(h_{o}\right)}$ (lemma 1). From theorem 3 , we know that the effective resistance between $u$ and $o$ in $N(G)$ is higher than the effective resistance between the corresponding nodes $\bar{u}$ and $\bar{o}$ in $N\left(\mathbf{Z}_{2}^{\left(h_{o}\right)}\right)$, where $N\left(\mathbf{Z}_{2}^{\left(h_{o}\right)}\right)$ is an electrical network constructed by assigning equal resistances $\sigma_{\min }^{2} I_{k}$ to every edge in $\mathbf{Z}_{2}^{\left(h_{o}\right)}$. Denote this effective resistance by $R_{\bar{u}, \bar{o}}^{\text {eff }}$ Therefore, $\Sigma_{u, o} \geq R_{\bar{u}, \bar{o}}^{\text {eff }}$. We now determine a lower bound on $R_{\bar{u}, \bar{o}}^{\mathrm{eff}}$.

From theorem 4, $R_{\bar{u}, \bar{o}}^{\text {eff }} \geq \Omega\left(\log \left(d_{\mathbf{Z}_{2}}(\bar{u}, \bar{o}) / h_{o}\right)\right) \sigma_{\min }^{2} I_{k}$. From lemma $1, h_{o} \leq 2(r+s) / s+1$ and $d_{\mathbf{Z}_{2}}(\bar{u}, \bar{o}) \geq$ $\sqrt{2}\left(d_{f}(u, o) / s-1\right)$. Therefore,

$$
\begin{array}{r}
\frac{d_{\mathbf{z}_{d}}(u, o)}{h_{o}} \geq \frac{\sqrt{2}\left(d_{f}(u, o) / s-1\right)}{2(r+s) / s+1} \geq \frac{\sqrt{2}\left(d_{f}(u, o)-s\right)}{2 r+3 s} \\
>\frac{d_{f}(u, o)}{5 r}
\end{array}
$$

where the last inequality follows from the fact that $s \leq r$ for a connected graph. Hence, $R_{\bar{u}, \bar{o}}^{\text {eff }} \geq$ $\Omega\left(\log \left(d_{f}(u, o)\right) / r\right) \sigma_{\min }^{2} I_{k}$. Since $\Sigma_{u, o} \geq R_{\bar{u}, \bar{o}}^{\text {eff }}$, we get the desired result.

Theorem 6: If $G$ is dense in $\mathbb{R}^{d}, 1 \leq d \leq 3$, and the conditions stated for theorem 5 are satisfied, the following statements hold:

1) If $G$ can be drawn in a dense manner in $\mathbb{R}$ with some $f$ with $\rho \geq 2 \gamma$, where $\rho$ and $\gamma$ are the minimum disconnected range and the maximum uncovered diameter in $G$ induced by $f$, then $\Sigma_{u, o} \leq \mathcal{O}\left(d_{f}(u, o) / \gamma\right)$.

2) If $G$ is dense in $\mathbb{R}^{2}$ with $\rho \geq 2 \gamma$, then $\Sigma_{u, o} \leq$ $\mathcal{O}\left(\log \left(d_{f}(u, o) / \gamma\right)\right) \sigma_{\max }^{2} I_{k}$

3) If $G$ is dense in $\mathbb{R}^{3}$ with $\rho \geq 2 \gamma$, then $\Sigma_{u, o} \leq$ $\mathcal{O}(1) \sigma_{\max }^{2} I_{k}$.

Moreover, the estimator variance has the following upper bound:

$$
\Sigma_{u, o} \leq d_{G}(u, o) \sigma_{\max }^{2} I_{k}
$$

where $d_{G}(u, o)$ is the graphical distance between $u$ and $o$ in $G$.

Proof. We will prove only the second statement (for $\mathbb{R}^{2}$ ) among the first three statements of the theorem, the statements for $\mathbb{R}$ or $\mathbb{R}^{3}$ can be proved in an analogous fashion.

In this case, we are interested in upper bounds on $R_{u, o}^{\mathrm{eff}}$ in $N(G)$. Since $G$ (and therefore $N(G)$ ) can be drawn in a dense manner in $\mathbb{R}^{2}$ with $\rho>2 \gamma$, the 2-D lattice $\mathbf{Z}_{d}$ 
can be embedded in $G$ (from lemma 2). If we construct the electrical network $N\left(\mathbf{Z}_{d}\right)$ from $\mathbf{Z}_{d}$ by assigning generalized resistances of $\sigma_{\max }^{2} I_{k}$ to each edge, we get an embedding of $N\left(\mathbf{Z}_{d}\right)$ in $N(G)$ where the resistances associated with the edges in $N\left(\mathbf{Z}_{d}\right)$ are greater than or equal to the resistances associated with the corresponding edges in $N(G)$. Hence, the effective resistances in $N(G)$ are lower than the corresponding effective resistances in $N\left(\mathbf{Z}_{d}\right)$ (from Generalized Rayleigh's Monotonicity Law). So, $\Sigma_{u, o} \leq R_{\bar{u}, \bar{o}}^{\mathrm{eff}}$ where $\bar{u}$ and $\bar{o}$ in $N\left(\mathbf{Z}_{2}\right)$ are the nodes corresponding to $u$ and $o$ in $G$.

The graphical distance between $\bar{u}$ and $\bar{o}$ in the lattice $\mathbf{Z}_{2}$ satisfies $d_{\mathbf{Z}_{2}}(\bar{u}, \bar{o}) \leq \sqrt{2}\left(d_{f}(u, o)+\gamma\right) / \gamma$ (from lemma 2 ). Therefore, from theorem 4 , with $h=1$, after some manipulation we get

$$
R^{\mathrm{eff}}(\bar{u}, \bar{o}) \leq \mathcal{O}\left(\log \left(d_{f}(u, o) / \gamma\right)\right) \sigma_{\max }^{2} I_{k} .
$$

Since $\Sigma_{u, o} \leq R^{\mathrm{eff}}(u, o)$, the desired result follows.

To obtain the upper bound in terms of the graphical distance $d_{G}$, consider the scenario when the graph $G$ is a tree and the node $u$ is at a graphical distance $d_{G}(u, o)$ from the reference in $G$. From Rayleigh's monotonicity law, the effective resistance in this case would be lower than or equal to that if all edge resistances were equal to the maximum possible value $\sigma_{\max } I_{k}$, which, with the electrical analogy gives us $\Sigma_{u, o} \leq d_{G}(u, o) \sigma_{\max }^{2} I_{k}$.

\section{CONCLUSIONS \& FUTURE WORK}

For two classes of graphs - civilized and dense in $\mathbb{R}^{d}, \quad 1 \leq d \leq 3$, we established asymptotic lower and upper bounds on the variance of a node variable $x_{u}$ 's optimal linear estimate as a function of the node's Euclidean distance from the reference node in a drawing of the graph. The main results are summarized in table I. Although the drawing of a graph is not unique, in practice this distancein-the-drawing can be directly related to the distance in physical space where the objects in the network are located. It is reasonable to expect that real sensor networks will satisfy the criteria for being civilized and hence the lower bounds derived here will hold in practice. The criteria for dense graphs under which the upper bounds hold may be conservative. Future research will concentrate on improving these bounds.

Although only order estimates were presented here in the interest of clarity, they can be easily made tighter by keeping all the terms in the derivation. Such bounds can be a useful tool in designing networks, since they establish fundamental limits on what level of estimation accuracy can be achieved as the size of network increases.

The question of how the variance of the optimal estimator for a node variable grows with distance from the reference is answered in terms of the Euclidean distance in a drawing of the graph. How the variance varies with graphical distance for graphs that are not trees is still an open question.

It may not be feasible in practice to directly employ the UMV estimator due to its centralized processing. However, our focus is on investigating fundamental limits of estimation accuracy, hence we consider the UMV estimator since it achieves the minimum possible variance among all linear estimators. Future research directions include considering constraints on energy consumption and routing complexity in developing estimation procedures.

\section{REFERENCES}

[1] P. Barooah and J. P. Hespanha. Linear optimal estimation from relative measurements: Electrical analogy and error bounds. Technical report, E.C.E. Dept., University of California, Santa Barbara, 2003. URL http: / / www . ccec. ece.ucsb. edu/ pbarooah/research_PB.html.

[2] J. Caffery and G. Stber. Overview of radiolocation in CDMA cellular systems. IEEE Communications Mag., 36(4):38-45, April 1998.

[3] P. G. Doyle and J. L. Snell. Random walks and electric networks. Math. Assoc. of America, 1984.

[4] T. Eren, W. Whiteley, A. S. Morse, P. N. Belhumeur, and B. D. Anderson. Sensor and network topologies of formations with direction, bearing and angle information between agents. In Proceedings of the 42nd IEEE Conference on Decision and Control, December 2003, 2003.

[5] A. Jadbabaie, J. Lin, and A. S. Morse. Coordination of groups of mobile autonomous agents using nearest neighbor rules. IEEE Transactions on Automatic Control, 48(6):988-1001, June 2003.

[6] A. Kalis and T. Antanakopolous. Direction finding in IEEE802.11 wireless networks. IEEE Transactions on Instrumentation and Measurement, 51(5):940-948, October 2002.

[7] R. Karp, J. Elson, D. Estrin, and S. Shenker. Optimal and global time synchronization in sensornets. Technical report, Center for Embedded Networked Sensing,Univ. of California, Los Angeles, 2003.

[8] J. M. Mendel. Lessons in Estimation Theory for Signal Processing, Communications and Control. Prentice Hall P T R, 1995.

[9] R. Moses and R. Patterson. Self-calibration of sensor networks. SPIE vol. 4743: Unattended Ground Sensor Technologies and Applications IV, 4743, 2002.

[10] N. Patwari, A. O. H. III, and M. Perkins. Relative location estimation in wireless sensor networks. IEEE Transactions in Signal Processing, 51(8):2137-2148, August 2003. 